\title{
Role of Diagnostic Laparoscopy in Penetrating Abdominal Trauma
}

\author{
FATIMA ABBASI ${ }^{1}$, MUHAMMAD KHURRAM ZIA ${ }^{2}$, FARHAN SIDDIQUE $^{3}$, ALI ADNAN $^{4}$, HINA TAHSEEN $^{5}$ \\ ${ }^{1}$ Consultant General Surgeon, Holy Family Hospital, Karachi \\ ${ }^{2}$ Associate Prof. of Surgery Liaquat College of Medicine and Dentistry/ Darul Sehat Hospital, Karachi \\ ${ }^{3}$ Assistant Prof. of Urology, Karachi Medical and Dental College \& Abbasi Shaheed Hospital, Karachi \\ ${ }^{4}$ Consultant Plastic Surgeon, Burns Centre, Civil Hospital, Karachi \\ ${ }^{5}$ Senior Registrar, Bahria University of Medical and Dental College and PNS Shifa Hospital, Karachi \\ Corresponding author: Fatima Abbasi, Email:dr_fatima_84@hotmail.com,Cell: 03343895138
}

\begin{abstract}
Background and Objective: Trauma remains the major cause of mortality and disability among young people across the world with penetrating trauma being a very common cause. Traditionally, penetrating abdominal trauma was managed with exploration. But now with the advent of minimally access surgery and advancements in laparoscopic expertise, more patients can be managed with minimally invasive methods. This approach can save many unnecessary laparotomies and large midline incisions. The role of laparoscopy in blunt abdominal trauma is well established. The aim of this study was to evaluate the role of diagnostic laparoscopy in penetrating abdominal injuries.

Methods: All the penetrating abdominal trauma patients presenting to the emergency department of Rawal Medical and Dental hospital from January 2019 till December 2020 for a period of 2years (a total of 102 patients) and who were hemodynamically stable, between the ages of 20 to 50 years of either gender were included in the study. All these patients had equivocal abdominal findings with no signs to suggest serious intraabdominal injury. These patients were prepared as standard for general anesthesia and diagnostic laparoscopy was performed. A predesigned performa was used for entering the patients' details and operative findings. All patients gave written informed consent in urdu. Main outcome measures were the conversion rate, missed injuries leading to reexploration.

Results: $85 \%$ of the patients were males with only $15 \%$ females. Mean age of the population was 38.7 years .Conversion to open was required in only $6.12 \%$ of the cases .Laparoscopy alone was sufficient for all other patients. In about $36 \%$ of the patients no intraabdominal injury was found. In rest $58 \%$ patients the surgeons were able to repair the injuries laparoscopically.18 patients had minor liver injury, 10 patients had minor hemoperitoneum $<100 \mathrm{ml}$ without any significant injury and 2 patients had single small bowel perforation which was repaired laparoscopically and cavity was irrigated. No patient had post operative complications of peritonitis due to missed injury or bleeding leading to re exploration.

Conclusion: Laparoscopy is a very effective procedure to deal with penetrating abdominal trauma patients who are stable and with equivocal abdominal findings without increasing risk of missed injury with minimal rates of conversion to open laparotomy if patients are selected vigilantly.

Keywords: Penetrating, Diagnostic Laparoscopy, Abdominal Trauma
\end{abstract}

\section{INTRODUCTION}

Penetrating abdominal trauma is a very common entity. The commonest cause nowadays is gunshot wounds and less common causes are stab wounds, road traffic accidents leading to broken glass injuries etc. The most common organs involved in decreasing frequency are small bowel ,large bowel, liver, spleen, vascular and retroperitoneum ${ }^{(1)}$. Conventionally, all penetrating trauma patients with suspicion of peritoneal penetration underwent exploration. This led to many unnecessary laparotomies. Recently, there is a shift towards laparoscopic evaluation of stable patients of penetrating abdominal trauma (2). Laparoscopy has been increasingly used in all forms of surgery nowadays. Its advantages in elective surgeries are well known and in many diseases like gall bladder diseases and recurrent bilateral inguinal hernias, it has long been accepted as a 'gold standard' therapeutic procedure. The role of diagnostic and therapeutic laparoscopy in blunt abdominal trauma is now well established ${ }^{(3)}$. Many surgeons are not comfortable managing penetrating trauma of abdomen laparoscopically. The main reason behind this being fear of missed intraabdominal injuries ${ }^{(4)}$ But now with emerging increased use of minimal access surgeries in all disciplines of surgery, trauma surgeons have also improved their technical skills in laparoscopy. Recent studies have shown very minimal rates of missed injuries which were very high in previous studies carried out in different centres ${ }^{(5)}$. This is largely due to growing surgical expertise and improvement in laparoscopic instruments. We conducted a prospective study in a busy urban trauma center to see whether laparoscopic surgery is safe in these patients.

\section{MATERIAL AND METHODS}

Between a period of 2years, the penetrating trauma patients having the following inclusion criteria were subjected to laparoscopic evaluation:

1 ages between $20-50$ years
2 equivocal abdominal findings

3 hemodynamically stable

4 no evidence of serious intraabdominal injury(anemia, generalized tenderness)

5 evidence of peritoneal penetration as suggested by local wound examination/exploration

A total of 102 patients met our inclusion criteria and diagnostic laparoscopy was performed.

Patients with peritonitis and vital instability were taken straight to $\mathrm{O} . \mathrm{R}$ for emergent exploration. Patients having any contraindication to laparoscopy were also excluded. Patients with penetrating trauma who had no abdominal signs and symptoms were admitted for C.T Scan. All the operating surgeons were of similar surgical expertise to minimize technical bias.

Surgical technique: Patients were given general anesthesia. Ports inserted were as follows:

$1: 10 \mathrm{~mm}$ in the umbilical region for telescope

2: $10 \mathrm{~mm}$ working port in right iliac fossa

3: $5 \mathrm{~mm}$ port in epigastrium

4: $1-2$ optional ports if required

Whole of the peritoneal cavity and retroperitoneum were examined. Findings noted and those cases where no injury was found were closed after thorough evaluation. In cases where liver or mental injury was the cause of hemoperitoneum, cavity was washed and re-examined for active bleeding and intracorporeal suturing was done to secure hemostasis where required. Small bowel injuries were also repaired. Those cases where repair was not possible laparoscopically, procedure was converted to open laparotomy.

\section{RESULTS}

Majority (85\%) of our study population were males. Mean age was 38.7 years. The intraoperative findings on laparoscopic evaluation are summarized in the Table 1: 
Table No 1: Intraoperative Findings on Laparoscopic Evaluation

\begin{tabular}{|l|l|l|}
\hline S no & Nature of injury & Percentage \\
\hline 01 & No intraperitoneal injury & $40 \%$ \\
\hline 02 & Diaphragmatic/multiple bowel injuries & $8 \%$ \\
\hline 03 & Isolated small bowel injury & $19 \%$ \\
\hline 04 & Liver injury & $23 \%$ \\
\hline
\end{tabular}

The small fraction of the patients i.e $8 \%$ (8 patients)had injuries which were not amenable to laparoscopic repair and were converted to midline laparotomy ( $p$-value 0.56$)$. All other patients had laparoscopic dealing of their injury( $p$ value 0.03 ).

The reasons of conversion are summarized in table 2 :

Table No 2: Causes of Conversion

\begin{tabular}{|l|l|l|l|}
\hline S no & Nature of injury & Number of patients & Percentage \\
\hline 01 & Diaphragmatic injury & 5 out of 8 & $62.5 \%$ \\
\hline 02 & Multiple bowel injury & 1 out of 8 & $12.5 \%$ \\
\hline 03 & $\begin{array}{l}\text { Retroperitoneal } \\
\text { hematoma }\end{array}$ & 2 out of 8 & $25 \%$ \\
\hline
\end{tabular}

Its evident that the commonest cause of conversion to open laparotomy was diaphragmatic injury followed by retroperitoneal hematoma and multiple small bowel injury respectively.Nonetheless the rate of conversion i.e $8 \%$ was small with $p$ value of $>0.05$ that is statistically insignificant.

\section{DISCUSSION}

Trauma is one of the leading cause of morbidity and mortality worldwide.It has been ranked as the number 1 casue of death in patients aged $20-45$ years by National trauma centre America.It is also $3^{\text {rd }}$ leading cause of death among all age groups ${ }^{(6,7)}$. Abdomen is the third commonest territory affected by trauma either blunt or penetrating ${ }^{(8,9)}$. Due to diversity of intraabdominal organs,trauma can present with a wide array of symptoms. Therefore,meticulous initial assessment and management is critical for better overall outcome.Controversy exists whether open or a laparoscopic approach is better fot those requiring some sort of intervention ${ }^{(10)}$.

Previously patients with penetrating abdominal trauma were managed by laparotomy and it was accepted as a Gold standard $^{(11)}$. With the advent of laparoscopy,the management of penetrating abdominal trauma patients who are hemodynamically stable has changed markedly.From big midline incisions to small port incisions and from days of admission to less than 24 hours admission if no injury is found. This shift towards the increased use of laparoscope in penetrating abdominal trauma is not sudden.Rather it's a gradual change over the decades of evolution of laparoscopic surgery ${ }^{(12,13,14)}$. Many studies have been carried out to ascertain the safety of this approach in stable penetrating trauma patients.And more and more trauma centers in the world are now making protocols and guidelines for stable trauma patients management by laparoscopy ${ }^{(15,16)}$.

Multiple controversies surround the indications, contraindications and correct method of diagnostic laparoscopy in penetrating abdominal trauma.In one study the main limitation to the use of laparoscopy and conversion to open laparotomy was retroperitoneal injuries ${ }^{(17,18)}$.In our study the main reason of conversion was diaphragmatic injury which compelled surgeon to repair it by openapproach. Retroperitoneal injuries were less common in our study,but when encountered open approach was preferred. Other reason for conversion in our study was multiple small bowel injuries which were compatible with other similar studies carried out to assess the limitations of conversion to open approach ${ }^{(18)}$.

One study carried out in 2017 on 192 patients of stable penetrating abdominal trauma without any overt indication of laparotomy showed almost similar results as our present study. The study population was divided into two groups of equal patients. One group underwent laparotomy and the other group underwent diagnostic+-therapeutic laparoscopy. The results were convincing, in the group of patients who underwent laparoscopy,the conversion rate was $11 \%$ that is slightly higher than our study. The commonest reason of conversion was multiple bowel injuries in contrast to our study.In patients undergoing laparotomy,the negative laparotomy rate was strikingly high i.e $38 \%$. This shows that overall, $77 \%$ of the total study population could have been managed by laparoscopy alone $^{(19)}$.

In another prospective study carried out by A Fabian et al,89 patients with penetrating abdominal trauma,therapeutic laparoscopy was used as a tool to find the indication of laparotomy and results were interesting. Overall, $49 \%$ of the patients had no abdominal penetration. Negative laparotomy rate was reduced to 1 $\%$ with this technique ${ }^{(20)}$. A recent metaanalysis in this regard is worth mentioning here which was published in International Journal of Surgery in 2016 (21). This analysis included 9 studies of which one was a randomized control trial and others 8 were observational studies. studies compared the stable penetrating trauma patients who either underwent laparotomy or diagnostic/therapeutic laparoscopy. The metaanalysis revealed the following key points as its conclusion:

there are many advantages of laparoscopy but the most significant one is avoidance of unnecessary non therapeutic laparotomies.

diagnostic laparoscopy of stable penetrating abdominal trauma patients is effective and safe alternative to open laparotomy .

missed injury is lower than commonly misperceived because of direct visualization of intraperitoneal organs.

most of the existing evidence on the use of diagnostic laparoscopy in penetrating abdominal trauma

$\sim$ more and more prospective studies and randomized control trials are needed to provide and support high level evidence in this regard.

In literature most of the studies carried out are descriptive and retrospective.This metaanalysis strongly recommended prospective randomized control trials for this purpose. A retrospective analysis based on a ten year data of trauma patients pusblished by John et al,addressed the laparoscopic intervention in stable trauma patients ${ }^{(22)}$. They concluded that with careful use of laparoscopy,about $70 \%$ of stable trauma patients can be prevented from traditional laparotomy. An additional advantage of laparoscopy was that incidental intraabdominal pathologies were also addressed in some patients.

The comparative mortality,morbidity and post operative complications were less than open laparotomy patients for obvious reasons. Another retrospective study conducted by LIN HF et al, conducted in 2010 concluded that laparoscopy patients had shorter operative duration,shorter hospital stay and lesser incidence of DVT and wound infcetion ${ }^{(23)}$. No procedure is without demerits. Apart from all these advantages,there are some complications specific to laparoscopy like gas embolism and tension pneumothorax. The later is more common in patients with diaphragmatic injury as pneumoperitonem enhances the severity of pneumothorax in already vulnerablehemithorax.

\section{CONCLUSION}

Laparoscopy is a very effective procedure to deal with penetrating abdominal trauma patients who are stable and with equivocal abdominal findings without increasing risk of missed injury with minimal rates of conversion to open laparotomy if patients are selected vigilantly.

Conflict Of Interest: None

\section{REFERENCES}

1 Villavicencio RT, Aucar JA, Analysis of laparoscopy in trauma. J Am Coll Surg. 2019;189:11-20

2 Short AR. The uses of celioscopy.BMJ. 2019;2:254-255

3 Sosa JL, Baker M, Puente I, et al. Negative laparotomy in abdominal gunshot wounds: Potential impact of laparoscopy. J Trauma. 2017;38:194-197

4 Ball CG. Current management of penetrating torso trauma: Nontherapeutic is not good enough anymore. Can J Surg. 2014;57:E36-43. 
$5 \quad$ Koto MZ, Matsevych OY, Motilall SR. The role of laparoscopy in penetrating abdominal trauma: Our initial experience. J LaparoendoscAdvSurg Tech A. 2015;25:730-6

6 O'Malley E, Boyle E, O'Callaghan A, Coffey JC, Walsh SR. Role of laparoscopy in penetrating abdominal trauma: A systematic review. World J Surg. 2013;37:113-22.

7 Matsevych O, Koto M, Balabyeki M, Aldous C. Trauma laparoscopy: When to start and when to convert? SurgEndosc. 2018;32:1344-52.

8 Kaufman $\mathrm{MH}$, Carswell A. Musket-ball and sabre injuries from the first half of the nineteenth century. Edinburgh: Royal College of Surgeons; 2003

9 Biffl WL, Leppaniemi A. Management guidelines for penetrating abdominal trauma. World J Surg. 2015;39:1373-80.

10 Kawahara NT, Alster C, Fujimura I et al (2009) Standard examination system for laparoscopy in penetrating abdominal trauma. J Trauma 67:589-595

11 Johnson JJ, Tabitha Garwe T, Alexander R, Raines AR, Joseph B Thurman JB, Sandra Carter S, Jeffrey S, Bender JS, Roxie M, Albrecht RM (2013) The use of laparoscopy in the diagnosis and treatment of blunt and penetrating abdominal injuries: 10-year experience at a level 1 trauma center. Am J Surg 205:317-321

12 Marwan A, Harmon CM, Georgeson KE, Smith GF, Muensterer OJ (2010) Use of laparoscopy in the management of pediatric abdominal trauma. J Trauma Injury Infect Crit Care 69(4):761-764

13 Sitnikov V, Yakuba A, Sarkisyan V, et al.The role of video-assisted laparoscopy in management of patients with small bowel injuries in abdominal trauma.SurgEndosc 2009: 23:125-129.

14 Leppaniemi A, Happianen R (2013) Diagnostic laparoscopy inabdominal stab wounds: a prospective, randomisedstudy.J Trauma 55:636-645.
15 Cherry RA, Eachempati SR, Hydo LJ et al (2015) The role of laparoscopy in penetrating abdominal stab wounds. SurgLaparoscEndoscPercutan Tech 15:14-17

16 Mahajna A, Mitkal S, Bahuth $\mathrm{H}$ et al (2009) Diagnostic laparoscopy for penetrating injuries in the thoracoabdominalregion.SurgEndosc 18:1485-1487

17 Miles EJ, Dunn E, Howard D et al (2014) The role of laparoscopyin penetrating abdominal trauma. J SocLaparoendosc Surg8:304-309.

18 Ertekin C, Yanar H, Taviloglu K et al (2011) Unnecessary laparotomy by using physical examination and different diagnostic modalities for penetrating abdominal stab wounds. Emerg Med J 22:790-794

19 Simon RJ, Rabin J, Kuhls D. Impact of increased use of laparoscopy on negative laparotomy rates after penetrating trauma. J Trauma. 2017;53:297-302

20 Fabion et al. Laparoscopy in the diagnosis of blunt and penetrating to the abdomen. Am J Surg. 2015;131:315-8.

21 Hajibandeh S, Hajibandeh S, Gumber AO, Wong CS. Laparoscopy versus laparotomy for the management of penetrating abdominal trauma: A systematic review and meta-analysis. Int $\mathrm{J}$ Surg 2016:34:127-36.

22 John, Whelan J, Brownlee $J$ et al (2005) The contribution oflaparoscopy in evaluation of penetrating abdominal wounds.J Am CollSurg 201:213-216

23 Lin HF, Wu JM, Tu CC et al (2010) Value of diagnostic andtherapeutic laparoscopy for abdominal stab wounds. World $\mathrm{J}$ Surg34:1653-1662. 